\title{
The first reported paramutation
}

Gregor Mendel's crossing experiments with pea plants led to the enunciation of the principles of inheritance in 1866 . William Bateson and Caroline Pellew's crossing experiments with pea plants, reported in 1915 (J. Genet. 5, 13-36), gave one of the first instances of nonconformity with these principles. The Bateson and Pellew paper was cited as recently as March 2021 in PLoS Genetics (https://doi.org/10.1371/journal.pgen.1009444). Few journals can boast of citation to a 106-year-old paper. The Journal of Genetics is like a Kohinoor that came the other way.

The 1915 and 2021 papers were connected by the theme of paramutation. Paramutation is an epigenetic switch that causes a gene to flip to a silenced state, which is then maintained in later generations. The active and silenced versions of the gene are called epialleles. As they share the same DNA sequence, they are genetically identical. But their DNA sequence is differentially modified, for instance differentially methylated, or its associated proteins are differentially modified. Hence, the difference is epigenetic. Significantly, a paramutated epiallele can induce secondary paramutation and silence its homologue in subsequent generations.

The PLoS Genet. study sought to understand how paramutation is established in the model plant Arabidopsis thaliana, and to identify parameters that influence it. The authors made crosses between plants bearing epialleles that resulted in resistance or sensitivity to an antibiotic in the growth medium. The Arabidopsis paramutation differs from that of maize and tomato (and peas) since it is evident only in the progeny of the $F_{1}$ hybrids (i.e., in the grand-offspring generation), and only in plants with a doubled chromosome set (i.e., tetraploid plants). Small RNAs (sRNAs) and RNA-directed DNA methylation played roles in silencing the resistance gene. The copy number ratio between active and silent epialleles and growth temperature were also important.

Many cultivated plant varieties, which are expected to breed true to type, sporadically throw small numbers of plants with a 'rogue' phenotype, which differs from the variety sown in characteristic ways. For example, tall plants in a dwarf variety, or plants with pods of the wrong shape. But in all other respects the rogues are identical to the normal variety. A variety can breed true for several generations and then throw a rogue. Bateson and Pellew investigated the emergence of the rogues in pea plants. Among other differences, the rogue peas made smaller seeds that were less sweet.

Since the rogues appeared to be 'untrue' to the type, seed growers took great effort to exterminate them. Thus, there were no large bodies of rogues that could provide a source for insects to pollinate the surrounding type. And it was unlikely that the rogues were contaminants, or represented the persistent recurrence of a recessive form from a heterozygote. Rogues bred true and had only rogue offspring. Notably, crosses between rogue and variety gave only rogue offspring. This result did not conform to Mendel's laws.

The epigenome of a rogue and its parental pea variety were examined recently (2017 Epigenomes 1, 6; https://doi.org/10.3390/ epigenomes1010006), and specific differentially methylated DNA sequences were found. For a subset of the sequences the differential methylation pattern was also conserved in the pollen grains, and presumably inherited in the progeny. These results hold the promise that progress in elucidating the molecular mechanisms underlying the emergence of rogues might be at hand.

E-mail: kas@cdfd.org.in.

Durgadas P. Kasbekar

Editor-in-Chief

Journal of Genetics 